A Guide for Many Authors: Writing Manuscripts in Large Collaborations

\author{
Hannah Moshontz* \\ University of Wisconsin-Madison \\ Charles R. Ebersole* \\ University of Virginia \\ Sara J. Weston \\ University of Oregon \\ Richard A. Klein \\ Tilburg University
}

$*=$ These authors co-led this project

Draft date: $01 / 28 / 21$

Manuscript status: Accepted at Social and Personality Psychology Compass

Author Note:

Corresponding Author: Hannah Moshontz, Department of Psychology, University of WisconsinMadison, 1202 W. Johnson St., Madison, WI 53715.

E-mail: hmoshontz@gmail.com 
Writing manuscripts collaboratively affords both opportunities and challenges: Collaborative papers can benefit from the expertise, perspectives, and collective effort of the group but can lack coherence or be produced inefficiently. When collaborations are large, involving ten or hundreds of researchers, there are more and different opportunities and challenges, like appropriately crediting the contributions of many people. This paper is a practical guide for authors writing collaborative manuscripts, particularly those working in large collaborations. We emphasize the importance of deliberate leadership and describe five general strategies that lead authors can employ to maximize opportunities and navigate challenges: care in recruiting the author team, care in crediting the author team, clear and frequent communication, organized materials, and deliberate and early decision-making. For each, we offer specific tips in line with these strategies (e.g., use collaboration agreements, leverage Open Science practices). We then suggest how lead authors can structure the writing and revising process to produce a coherent manuscript and offer tips for submitting papers and responding to peer-reviews. A repository of resources for people writing manuscripts in collaborations is available at osf.io/dzwen 
A Guide for Many Authors: Writing Manuscripts in Large Collaborations In collaborations, independent researchers contribute their skills, expertise, and resources to one research project. In manuscripts that describe collaborative projects, independent researchers contribute their words, perspectives, and insights to one paper. Research in psychological science has historically been conducted by small teams of two or three authors (e.g., Guimera, 2005), but large-scale collaborative projects involving tens or hundreds of researchers at different institutions are becoming more common in psychology and other sciences and so too are papers written by tens or hundreds of authors (Wang et al., 2016; Wuchty et al., 2007). These papers describe new studies (e.g., Ebersole et al., 2016; Klein et al., 2014, 2018), meta-scientific research (e.g., Silberzahn et al., 2018), secondary analyses (e.g., Graham et al., 2017), or present non-empirical arguments (e.g., Benjamin et al., 2018; Lakens et al., 2018; Open Science Collaboration, 2012). All kinds of manuscripts can be improved by having many authors involved. The more authors writing a paper, the more likely it is that each section is grounded in specific expertise. The more authors revising a paper, the more opportunities there are to catch factual errors, typos, flawed logic, biases, and clumsy sentences. Just as many researchers can work together to produce research that is more ambitious and impactful than research produced by individuals or small teams (Newman, 2004; Uhlmann et al., 2019; Wuchty et al., 2007), they can work together to write manuscripts that similarly benefit from the input of many.

Though more authors bring unique opportunities to manuscripts, they also bring challenges that, if not managed, can reduce the marginal value of additional collaborators (Petersen et al., 2012). Coauthors' differing perspectives and opinions can undermine the coherence of the final paper, and the writing process may be slow and disorganized. Unmet 
expectations about authorship and crediting can cause interpersonal conflict and harm (Smith et al., 2019) and delay the publication process. In addition, large collaborations face problems that are not present in collaborations with smaller teams. Some challenges are simply a function of scale; as an author list grows, some ordinary tasks become harder. For example, integrating feedback on drafts takes more time and care due to the many perspectives being considered. The more people there are, the more opinions and writing styles there are, and the more likely it is that people's preferences and assumptions about how to write the paper will not align. In large collaborations, logistical complexities can slow the writing process and although people often begin a writing project full of enthusiasm, ideas, and focus, long delays and missed deadlines can deflate this motivation.

Other problems that can arise during the writing process in large collaborations are not merely of scale, but are entirely new problems that require new kinds of solutions. For example, diffusion of responsibility can cause some tasks, like proofreading supplemental material, to fall through the cracks or be done haphazardly when proofreaders take less personal responsibility for finding errors, assuming someone else will catch errors they miss (Forsyth et al., 2002). As a result of new problems that can emerge in large collaborations, familiar writing and publishing conventions do not always work. For example, authorship order is less effective at signaling relative contributions when there are twenty or two hundred contributors rather than two or three (Sauermann \& Haeussler, 2017).

Although these and other challenges are inherent to writing manuscripts with many authors, they can be overcome or reduced in practice. In addition, the unique opportunities of large collaborations can be leveraged so that the collective expertise and knowledge of the group is not wasted. This paper is a practical guide to writing manuscripts with many authors, based on 
our personal experiences leading and contributing to large, collaborative manuscripts. Empirical research on team science offers general recommendations on collaborating that are broadly consistent with those offered here (e.g., the need for clear communication and strong leadership; Bennett \& Gadlin, 2012; Hall et al., 2018). We cite empirical evidence where it is relevant, but the relevance of research on team science to our guide is limited because team science research typically focuses on outcomes of a group's productivity (e.g., citation counts, Hall et al., 2018) rather than on outcomes of a group's writing process (e.g., number of errors in a final report, writing clarity).

In this guide, we emphasize the importance of deliberate leadership, recommend five general strategies and numerous specific tips for lead authors, then describe detailed strategies for structuring the writing process and navigating the peer-review process. We also offer a repository of resources available at osf.io/dzwen.

\section{Deliberate Leadership}

An essential component of writing manuscripts in collaborations is deliberate leadership. In order to leverage opportunities and navigate challenges of writing papers in large collaborations, people's knowledge, expertise, and effort must be directed. We recommend that someone serve as the lead author, taking explicit responsibility for the manuscript and ensuring its completion by handling important tasks or delegating them to capable others. In some cases, it may make sense for the lead author role to be shared by multiple people who have explicitly agreed to specific individual responsibilities.

Whether the role is shared or assumed by one person, we recommend that lead authors employ five general strategies, each of which we discuss in detail: 1) care in recruiting a coauthor team, 2) care in crediting the coauthor team, 3) clear and frequent communication, 4) 
organized materials, and 5) early and deliberate decision-making. For each, we offer specific tips for lead authors.

\section{Strategies for Writing Manuscripts in Large Collaborations}

\section{Strategy 1: Care in Recruiting a Coauthor Team}

Who is listed as a coauthor on a final manuscript is determined by how (and who) you recruit to join the coauthor team. Recruiting coauthors through collaboration networks (e.g., StudySwap, Chartier et al., 2018; ManyBabies, Frank et al., 2017; Many Primates, Many Primates et al., 2019; the Psychological Science Accelerator, Moshontz et al., 2018) or through large forums (e.g., society listservs, Twitter) will likely result in a more diverse coauthor team (e.g., with respect to geographic distribution, expertise) than recruiting through personal networks. Diversity of a research team can benefit research quality and outcomes (Cheruvelil et al., 2014) and may also benefit the quality of the final manuscript.

The costs of conducting research and writing a paper with many other people should be justified by specific benefits of having a large team. Before recruiting a large team, consider whether and why a large collaboration is needed for your project.

Tips for lead authors. When recruitment begins, give consideration to the final manuscript. Large-collaborations that collect new data (e.g., the Many Labs series, Ebersole et al., 2016; Klein et al., 2014, 2018) often put out general calls to researchers, with all that are able to collect the minimum amount of data eligible to join. Some may have deep substantive knowledge about the topic of the project; others may not. How people are recruited impacts how much rank-and-file collaborators can or will contribute to primary writing of the manuscript. If there are areas of the envisioned paper where you have less knowledge, engage in targeted recruitment of experts (e.g., researchers with deeper knowledge of a particular theory or 
statistical technique). These targeted recruits may have a more involved role during specific phases of your project or drafting parts of the manuscript. However, avoid inviting so many people that some collaborators aren't contributing to the final manuscript. Each author on the final paper should have contributed in specific ways, such as informing the study design, overseeing data collection at a collection site, contributing to data analysis and interpretation, or helping write the initial draft of the manuscript or a particular section of the manuscript (Committee on Publication Ethics, 2004).

\section{Strategy 2: Care in Crediting the Coauthor Team}

How people are credited for their contributions can cause many problems in large collaborations (Holcombe, 2019; Sauermann \& Haeussler, 2017; Smith et al., 2019). Large collaborations are new to psychological science, and traditional methods of crediting via assigning authorship do not always work in large teams. Haphazard approaches to authorship in large collaborations, like signaling relative contributions through authorship order and having contributors decide whether their contributions merit authorship, can result in inconsistent crediting, people receiving credit for work they didn't do, and people failing to receive credit for work they did do. Such issues with crediting can cause harm to coauthors and their careers and can stall the publication process.

Lead authors who are careful in how they approach crediting can help all coauthors feel they have been fairly and appropriately credited on large collaborative projects. There are two related dimensions of credit: how authorship is allocated and how individuals' contributions are described. The best strategy for allocating authorship is to establish clear and specific authorship criteria before recruiting coauthors, and to encourage feedback or discussion about the criteria from potential coauthors. People have different opinions about what contributions merit 
authorship (Patience, Galli, Patience, \& Boffito, 2019), and authors may be differently benefited by the chosen method of crediting contributions depending on their institutional or national contexts. Communicating how authorship will be awarded at the start of a project gives people the opportunity to avoid working on collaborations that they don't feel offer fair credit or to find a solution that fits their credit preferences better (e.g., agreeing to take on an additional role that confers different credit). The best strategy for describing individuals' contributions is an explicit one. Rather than rely on authorship order to indicate contributions, contributions can be listed on the final manuscript (Allen et al., 2014, 2019; Holcombe, 2019).

Tips for lead authors. Outline how you will award authorship before people have made substantial contributions to the project. Create a collaboration agreement that applies to all collaborators and describes how authorship will be awarded and structured. There are several approaches to structuring authorship in large collaborations. One approach is to group authors into coarse, ordered categories based on their contributions (e.g., Moshontz et al., 2018). Within the categories, authors can be listed in an arbitrary order (e.g., alphabetically). Another approach is to list the name of the collaborative group as the sole author of a paper and provide individual names and contributions in an author's note (e.g., Open Science Collaboration, 2012, 2015). This second approach is more consistent with a contributorship model than with a traditional authorship model (Holcombe, 2019).

On the final manuscript, credit should be given in accordance with any existing collaboration agreement. The manuscript can list the specific contributions of all authors and any contributors who are not authors, ideally using standardized language (Brand et al., 2015). For example, the CRediT system recognizes 14 broad forms of contributorship and provides language for describing collaborator roles. One important advantage of a standard 
contributorship system is that it supports crediting roles like programming that are not always formally acknowledged (Allen, O’Connell, \& Kiermer, 2019).

\section{Strategy 3: Clear and Frequent Communication}

Large collaborations often include people with different amounts of expertise and experience, who are working in different personal contexts, institutions, geographic locations, cultures, and nations, who may use different languages, academic calendars, and time zones. These characteristics of large teams create the need for clear and frequent communication during the writing process. In small collaborations, authors often have existing systems or can correctly make assumptions about what role to take in the writing process and where to find materials, including the most recent draft of a manuscript. In contrast, people working in large collaborations may not know whether or how they are to contribute (e.g., providing new content, editing for clarity, copy-editing, fact-checking), and such information gaps can be hard to identify and resolve because the barrier for asking clarifying questions is higher (e.g., it often requires someone to send an email). Furthermore, communication in large collaborative teams is often written (e.g., via emails or comments on shared documents) and directional, rather than conversational. Thus, in large collaborative projects, lead authors must explicitly communicate a lot of information, and do so often and as clearly as possible.

How lead authors communicate during the writing process will determine how efficiently and well the manuscript is written. Poor communication may result in errors in a manuscript's content, delays in the writing and publication process, and may inconvenience or even offend coauthors. At any point during the writing process in a project with effective communication, all authors will know what is happening with the project and what the broad timeline for completion is. During times when action is requested of authors, lead authors can prevent wasted time and 
effort by making specific requests and providing clear and thorough instructions. In the absence of clear communication, people may contribute in ways that are moot or redundant, or they may not make contributions that would have benefitted the paper.

Tips for lead authors. Communicating with a large team can be very time-consuming for lead authors. One way to communicate efficiently is to reduce the number of one-on-one communications and rely heavily on mass communication. We recommend providing regular answers to the three questions most likely to be asked by collaborators: (1) What should I be doing right now? (2) How should I do it? (3) When does it need to be done by? The answers to these three questions can guide your collaborators through much of the project without you having to direct them individually. Mass communications can make collaboration efficient by providing these answers and the necessary resources to carry out the current steps of the project.

As an example, consider the process of collecting feedback on an initial draft of a manuscript. In order to effectively give feedback, collaborators will need to know where they can access the current draft of the manuscript, what you would like them to edit or focus on (e.g., are there particular sections that need closer review), how you would like them to make edits (e.g., using Suggesting mode in a shared document), and when the draft will be closed to new revisions. A mass email to all coauthors that answers those questions will allow your collaborators to immediately begin giving feedback. As a general rule of thumb, if you're receiving multiple responses asking for clarification, your original email was likely not sufficient. Instructions that require clarification not only make you spend time clarifying, often by responding to individual emails, but they also inconvenience the collaborator who must wait for you to respond before contributing. 
Although mass communication is efficient, it can be overwhelming. Send mass communications to the people they pertain to, rather than to all coauthors by default. Strive to keep everyone informed and up-to-date, while avoiding overtaxing your collaborators' bandwidth such that requests or details get overlooked. Compose communications in ways that will help strike this balance: state the message's intended audience and purpose within the first few sentences, indicate whether or not a response is needed (and if so, by whom and when), make important information easy to find (e.g., list important deadlines clearly and redundantly), and optimize communications for search (e.g., use the same project name in all email subject lines). Choose a mode of communication that matches your purpose. Some communication methods are better for making announcements or documenting information that might be referenced later (e.g., email, an OSF page), other communication methods are better for exchanging information (e.g., Slack) or having discussions (e.g., comments of a google doc, video chat). Confirm that recipients are receiving your communication; sometimes emails or listservs with lots of recipients get flagged as spam. For email communications, a simple fix is to CC yourself.

Finally, provide a way for coauthors to communicate with you. Invite your collaborators to ask questions and provide them with a means of preferred communication when it is ambiguous (e.g., who on a multi-person lead author team they are to contact, whether you would like them to ask clarifying questions on a public forum). Inviting communication from coauthors will help you identify and resolve miscommunications, and it will also help you respect your coauthors' preferences and constraints. Collaborators' different institutional, geographic, and cultural contexts can influence their availability and needs during the writing process, including when in the day and academic year they are available, what they are able to contribute, and the 
kinds of work that they are rewarded for. For example, universities in different countries operate on different academic calendars and individual researchers may adhere to cultural norms that affect their availability (e.g., it is common for individuals in Western Europe to go on vacation in August). These differences are likely to be wide ranging, and it is therefore difficult to anticipate all of them. Invite information about constraints and preferences that affect the writing process to avoid making inconsiderate assumptions, decisions, or requests (e.g., setting a deadline during a holiday).

\section{Strategy 4: Organized Materials}

There are often many materials associated with writing a manuscript. Organizing these materials well is essential to an efficient writing process and to producing an error-free manuscript. When materials are inaccessible or practically inaccessible because they are disorganized, people may end up not contributing, contributing redundantly, or contributing inefficiently. For instance, when authors do not know which version of a manuscript is the most current, they may make revisions on an older draft that are either moot or that require additional reconciliation effort to be useful.

To contribute usefully, coauthors must not only know what they should be doing, how they should do it, and when they need to do it, but they must also have access to materials. Thus, in an efficient collaborative writing process, materials are organized and accompanied by clear, redundant documentation. Organized materials not only help authors contribute quickly and to their fullest potential, it will also reduce the communication burden on lead authors. For example, if a detail-oriented author wants to check that the reported method is precisely correct, and they can easily access the version of the survey that was administered, they will not need to email the lead authors before working. 
Tips for lead authors. Make sure that all materials associated with a project are 1) stored in a shared location that all coauthors can access, 2) documented and/or labeled clearly so that they are understandable, and 3) stored in non-proprietary file formats that any author can open and use. For documents that are updated by multiple people over time, store the most current version in a shared location with version control so that people can work simultaneously on the most current version. Use folders to organize different kinds of materials, such as analytic scripts or data, and label them with clear and short names. Store proprietary materials and materials that will not be made public separately from materials that can or will be made public, and clearly describe whether and why certain materials cannot be shared. Choose a single platform to share materials and documentation. There are many platforms available -- the Open Science Framework, Dropbox, Google Drive, Github, etc. Generally, we recommend choosing a platform that includes version control, which all of the platforms listed above do.

An effective way to make sure that your collaborators have everything they need is to plan for all study materials, data, and scripts to be shared with the public. Although it is not always possible to share everything publicly, documenting and storing all study materials so that they can be shared with the broader scientific community will also make sharing these materials with your coauthors easy. If you will share your study materials publicly, choose a platform for internal organization that will allow you to make all or some of your materials public later, such as OSF or Github.

\section{Strategy 5: Early and Deliberate Decision-Making}

Decision-making becomes increasingly difficult as more stakeholders become involved, because people are more likely to have conflicting opinions about the manuscript's content, writing, or dissemination (e.g., where to submit the manuscript for publication). Also, because 
large teams have more varied values, incentives, and preferences about the publication process, it can be hard to find one decision that satisfies all authors. Unlike in smaller and more personally familiar author teams, conflicts in large teams cannot always be resolved through group discussion and consensus, and there need to be other mechanisms for reaching decisions. If care is not taken, the final manuscript and how it is published may reflect arbitrary and suboptimal decisions or may fail to reflect author opinions appropriately. For example, the final manuscript may not reflect the opinions that have the most merit if decisions are made with deference to the most senior authors or the most confident authors.

Lead authors can reduce the difficulty of decision-making by being deliberate about when decisions are made and who is involved in the decision process (e.g., Asencio et al., 2012). As one example, consider the process of choosing the topic for a large empirical collaboration. One strategy is for the lead author to narrowly define the topic before recruiting collaborators. For instance, they may want to conduct a multi-site test of a theory and have a particular study in mind. Another strategy is for the lead author to recruit potential collaborators for the project based on its broad goals and then solicit opinions from the group about the specifics (e.g., which particular study to replicate). The former strategy involves making crucial decisions among a small team, earlier in the process; the latter involves many individuals and occurs later. Another decision lead authors face is where to submit the report for publication. Like specifying the methods, this decision could be made by the lead author before recruiting collaborators, by the entire team before the project begins, or by the entire team after the research is done and before the report is written. In these examples and in all decisions lead authors face, there are multiple justifiable approaches. What we'd like to emphasize is that timing and who is involved will affect how easy the decision-making process is and, often, what decision is reached. Generally, 
lead authors can make decisions more quickly than a large team of collaborators. If there are fewer opinions, there is less to reconcile before making a decision. However, considering fewer opinions may make for a less optimal decision ultimately.

Tips for lead authors. Make decisions as early as is feasible. If an important decision is left for late in the process, be deliberate and transparent about how the decision will be made. Consider which decisions are best made collectively, and which decisions you would prefer to make independently. Be clear about this distinction: Only ask for opinions when you truly want them and will consider them. Inviting opinions broadly when you don't want them might result in a tough choice between abandoning your desired outcome or needing to overrule the opinions of your collaborators, which can reduce morale and potential contributions. Make independent decisions early, ideally early enough that you can invite collaborators based on those choices.

When writing empirical papers, consider leveraging transparent practices to help guide early and deliberate decision-making. Open, transparent research practices can benefit the scientific community and accelerate the acquisition of knowledge (Munafò et al., 2017; Nosek \& Bar-Anan, 2012) and they can also personally benefit you, particularly when managing many authors. Preregistration requires you and your team to make analytic choices early and may help to avoid debates about which analyses are most appropriate once the data are known. The Registered Reports publishing model (Chambers, 2013; Hardwicke \& Ioannidis, 2018) can be particularly beneficial to writing large collaborative manuscripts. In this model, authors submit a paper describing a project's rationale, methods, and planned analyses for peer reviewed prior to data collection. Papers can then receive in-principal acceptance from a journal contingent on carrying out the planned analyses, which necessarily front-loads many decisions about the writing and submission process. The Registered Reports model also naturally distributes and 
structures the writing process in ways that can be logistically beneficial. Focusing on different aspects of the paper at different times prevents coauthors from being overwhelmed by the amount of text to review and lead authors from being overwhelmed by the amount of feedback to integrate. As an additional benefit to the project, an in-principle acceptance can benefit recruitment; individuals may be more inclined to join as collaborators if they know the paper is already provisionally accepted.

\section{Tips for Structuring the Writing Process}

Many-authored papers have the strength of leveraging a vast amount of expertise. However, that expertise can be difficult to distill into a single coherent manuscript (that people will enjoy reading). Creating a coherent paper will require that lead authors aggregate many different opinions put forth by coauthors, or present multiple arguments in a coherent way, all while presenting them with a consistent writing style and flow. To achieve coherence and consistency, we recommend that lead authors structure the writing process, being deliberate about how much and which pieces of the paper they draft vs. delegate and directing the focus of revisions. In this section, we provide suggestions for lead authors related to structuring the writing process. Figure 1 depicts a useful process for writing manuscripts with many authors. We describe this process and stage-specific tips in further detail:

Initial draft. Although the entire team will revise the paper, someone must be in charge of creating the first draft of any given section. Barring total rewrites, this first draft will be the groundwork that others build upon. Although a first draft can be written quickly by a broader team, the more voices there are contributing new content, the greater the chance that the draft will be disjointed. Different people have different writing styles, and papers that rapidly switch from one voice to another can be difficult to read. In general, it may be best for you to take 
responsibility for creating an initial draft that all coauthors help revise. Another strategy is to delegate specific sections (e.g., Methods, Results) of the paper to other authors. When pursuing the latter strategy, we recommend allocating entire sections that are more narrative (e.g., the Discussion) and delegating smaller pieces only when they involve reporting (e.g., the sample characteristics section of the Method). Breaking apart pieces of the paper that are more narrative in nature, such as the Discussion, is more likely to lead to disjointed style.

Figure 1. Recommended steps of the writing process with many authors.

Soliciting and consolidating feedback. Another challenge to writing in large collaborations is incorporating the suggested revisions of many coauthors. In general, you want to encourage your coauthors to give feedback on the manuscript. Authors (especially junior ones) may feel intimidated to give feedback in such large teams. Similarly, if an author's suggested

Lead author(s) write initial draft

- Include major arguments

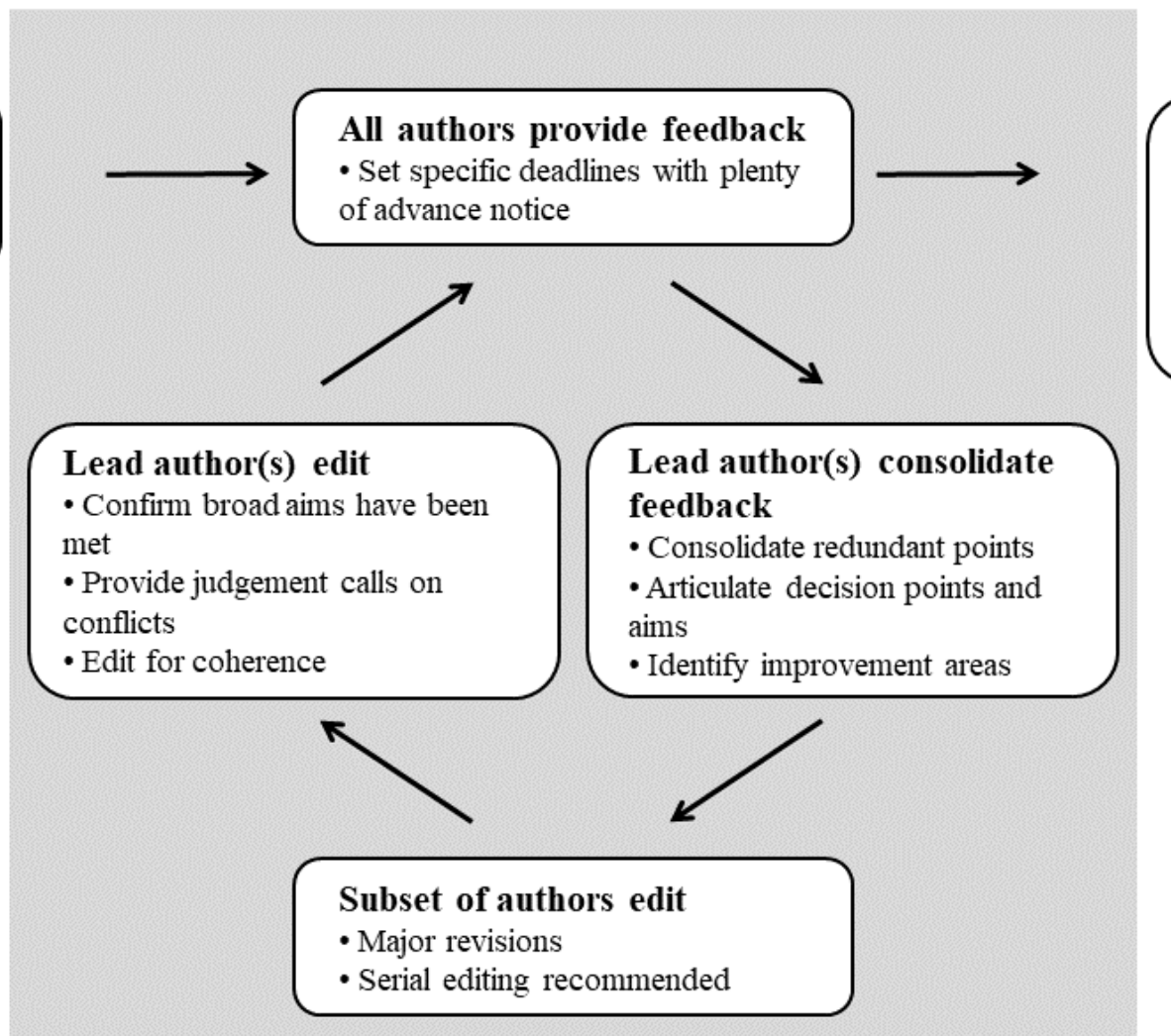

Iterate through these steps as required
Lead author(s) finalize draft

- Focus on comments related to clarity and factual correctness - Finalize decisions on conflicts with aim to find compromise 
revisions are repeatedly dismissed, they may become discouraged and stop offering feedback. Both of these situations may result in authors limiting themselves in what they suggest, which is a disservice to the final paper.

To help reduce this problem, we recommend providing a lot of structure when seeking revisions. For instance, if you share drafts of your paper in stages, request revisions on only the newly drafted section. Requesting revisions on just the newly drafted sections gives direction to members who may feel more intimidated and may discourage others from suggesting edits to sections you consider fully drafted. If you have specific concerns about the paper or places where you think it could be strengthened, mention those when sharing the paper. Again, making clear requests focuses the attention of your collaborators and can encourage more participation.

Overall, you want to encourage your collaborators to engage in the writing process with you while also limiting the number of times you need to reject their suggestions.

An effective workflow for revising a draft in a large collaboration is one in which you and a small subset of coauthors make major, iterative edits in response to feedback from the larger team. Especially when major edits are needed, having a group of one to five people make complete passes of the document can help maintain coherence and consistency. The gray box of Figure 1 depicts the suggested steps of soliciting and consolidating feedback. After you, or you and others, produce an initial draft and all authors provide feedback, you can iterate through a process in which a small group of authors makes edits. First, consolidate redundant points, identify conflicts and summarize main aims of the revision, identifying any specific areas that need improvement. With this information, a subset of authors can make major revisions to the manuscript, ideally working serially. After all members of this small group have made revisions, you can confirm that major decisions have been made or reach decisions based on the input you 
received and edit the paper for coherence before sending it back to all authors for more feedback. Cycling through this process until no major revisions are needed is an efficient way to produce coherent and thorough revisions.

Finalizing the draft. Ultimately, you must decide when the draft is ready to be submitted. Finalizing the draft will require making any edits related to clarity and factual correctness and resolving conflicts, with an aim to find compromise. Communicate when the final round of revisions is taking place, requesting only minor comments, such as checking for grammatical errors and factual correctness.

\section{Tips for Navigating the Peer-Review Process}

Submitting a manuscript and responding to reviews involves many procedural steps. These are amplified with large-scale collaborations. The same general strategies that benefit the writing process benefit the peer-review process, so we recommend that lead authors continue to communicate frequently and clearly, be organized, and make early and deliberate decisions about submissions and revisions. In addition, specific strategies can help make the peer-review process more efficient.

Submitting the manuscript. Submitting a manuscript with tens or hundreds of coauthors is tedious and time consuming, and the lead author is responsible for navigating the process without making any errors. Approaching the task systematically will save time and prevent errors. Before the paper is finished, assess what information you will need from each author in order to submit the manuscript. Required author information typically includes authors' publishing name, affiliation, department, and some contact information (e.g., email address). Optionally, many journals use ORCID (https://orcid.org/), which is a digital identification system for researchers. Collecting and reporting ORCID iDs is a good way to make sure that you have 
correctly identified all authors of a large collaborative manuscript. In addition to providing information about themselves, all authors will also have to approve of the final draft and disclose any Conflicts of Interest. A brief survey is an easy way to collect and consolidate these pieces of information during the revision process. Moreover, the journal may accept the survey results in lieu of manual entry of co-authors information through the submission portal. On very large projects, we recommend that you ask authors to create or update personal accounts in the journal submission system and then provide their journal-associated email. Having authors enter their own information and linking to that information with an email address can prevent errors and save you hours of manual entry.

Making revisions. After being submitted and reviewed, most papers will require major revisions. Structuring the revision process thoughtfully allows all authors to be informed of and involved in the revision process. If too many authors are trying to revise the paper simultaneously, revision requests may get overlooked and uncoordinated changes may disrupt the paper's coherence and consistency. For a smooth revision in response to peer-reviews, carefully structure coauthors' contributions. We recommend that as lead author you attempt to fully address reviewer concerns before soliciting feedback from the entire team. Before sharing the revised manuscript with collaborators, help collaborators understand the revisions. Provide a document of reviewer comments that documents and explains your revisions or responses to each. Use track-changes on the revised version of the manuscript to identify new text, which will help your collaborators focus their feedback on the changes. It may be most efficient for collaborators to limit feedback to just the revised portions of the manuscript. Inviting unnecessary revisions will take time and making too many unnecessary revisions may make for a confusing response to reviewers. 


\section{Conclusion}

Writing excellent papers with many authors often requires considerable work. Lead authors need to establish and communicate terms of collaboration, communicate clearly and frequently with their coauthors, organize materials, and make decisions deliberately and early. This additional work, compared to papers written by smaller teams, is more than made up for by what it can enable, as evidenced by the generally high quality of many-author publications (Hall et al., 2018; Uhlmann et al., 2019; Wuchty et al., 2007). Collaborative research projects often benefit from more thorough and creative consideration of the research questions, methods, and interpretations, and the resulting manuscript frequently covers a topic of broad interest. Moreover, as psychological science moves towards the adoption of larger and more diverse samples to support rigorous and generalizable research, large, multi-site, and many-author publications will continue to become more common. 


\section{References}

Allen, L., O'Connell, A., \& Kiermer, V. (2019). How can we ensure visibility and diversity in research contributions? How the Contributor Role Taxonomy (CRediT) is helping the shift from authorship to contributorship. Learned Publishing, 32(1), 71-74. https://doi.org/10/gf4jjv

Allen, L., Scott, J., Brand, A., Hlava, M., \& Altman, M. (2014). Publishing: Credit where credit is due. Nature, 508(7496), 312-313. https://doi.org/10/sdf

Asencio, R., Carter, D. R., DeChurch, L. A., Zaccaro, S. J., \& Fiore, S. M. (2012). Charting a course for collaboration: A multiteam perspective. Translational Behavioral Medicine, 2(4), 487-494. https://doi.org/10/ghttbm

Benjamin, D. J., Berger, J. O., Johannesson, M., Nosek, B. A., Wagenmakers, E.-J., Berk, R., Bollen, K. A., Brembs, B., Brown, L., Camerer, C., Cesarini, D., Chambers, C. D., Clyde, M., Cook, T. D., De Boeck, P., Dienes, Z., Dreber, A., Easwaran, K., Efferson, C., ... Johnson, V. E. (2018). Redefine statistical significance. Nature Human Behaviour, 2(1), 6-10. https://doi.org/10/cff2

Bennett, L. M., \& Gadlin, H. (2012). Collaboration and team science: From theory to practice. Journal of Investigative Medicine, 60(5), 768-775. https://doi.org/10.2310/JIM.0b013e318250871d

Brand, A., Allen, L., Altman, M., Hlava, M., \& Scott, J. (2015). Beyond authorship: Attribution, contribution, collaboration, and credit. Learned Publishing, 28(2), 151-155. https://doi.org/10/gc6v3m

Chambers, C. D. (2013). Registered Reports: A new publishing initiative at Cortex. Cortex, 49(3), 609610. https://doi.org/10/gf9dch

Chartier, C. R., Riegelman, A., \& McCarthy, R. J. (2018). StudySwap: A Platform for Interlab Replication, Collaboration, and Resource Exchange. Advances in Methods and Practices in Psychological Science, 1(4), 574-579. https://doi.org/10/gf5svf

Cheruvelil, K. S., Soranno, P. A., Weathers, K. C., Hanson, P. C., Goring, S. J., Filstrup, C. T., \& Read, E. K. (2014). Creating and maintaining high-performing collaborative research teams: The importance of diversity and interpersonal skills. Frontiers in Ecology and the Environment, 12(1), 


\section{1-38. https://doi.org/10/f52rtd}

Committee on Publication Ethics. (2004). The COPE report 2003: Annual report of the Committee on Publication Ethics. https://publicationethics.org/annualreport/2003

Ebersole, C. R., Atherton, O. E., Belanger, A. L., Skulborstad, H. M., Allen, J. M., Banks, J. B., Baranski, E., Bernstein, M. J., Bonfiglio, D. B. V., Boucher, L., Brown, E. R., Budiman, N. I., Cairo, A. H., Capaldi, C. A., Chartier, C. R., Chung, J. M., Cicero, D. C., Coleman, J. A., Conway, J. G., ... Nosek, B. A. (2016). Many Labs 3: Evaluating participant pool quality across the academic semester via replication. Journal of Experimental Social Psychology, 67, 68-82. https://doi.org/10.1016/j.jesp.2015.10.012

Forsyth, D. R., Zyzniewski, L. E., \& Giammanco, C. A. (2002). Responsibility diffusion in cooperative collectives. Personality and Social Psychology Bulletin, 28(1), 54-65. https://doi.org/10/dbsdv6

Frank, M. C., Bergelson, E., Bergmann, C., Cristia, A., Floccia, C., Gervain, J., Hamlin, J. K., Hannon, E. E., Kline, M., Levelt, C., Lew-Williams, C., Nazzi, T., Panneton, R., Rabagliati, H., Soderstrom, M., Sullivan, J., Waxman, S., \& Yurovsky, D. (2017). A collaborative approach to infant research: Promoting reproducibility, best practices, and theory-building. Infancy, 22(4), 421-435. https://doi.org/10/gf8qch

Graham, E. K., Rutsohn, J. P., Turiano, N. A., Bendayan, R., Batterham, P. J., Gerstorf, D., Katz, M. J., Reynolds, C. A., Sharp, E. S., Yoneda, T. B., Bastarache, E. D., Elleman, L. G., Zelinski, E. M., Johansson, B., Kuh, D., Barnes, L. L., Bennett, D. A., Deeg, D. J. H., Lipton, R. B., ... Mroczek, D. K. (2017). Personality predicts mortality risk: An integrative data analysis of 15 international longitudinal studies. Journal of Research in Personality, 70, 174-186. https://doi.org/10/gcf6sn

Guimera, R. (2005). Team assembly mechanisms determine collaboration network structure and team performance. Science, 308(5722), 697-702. https://doi.org/10/brgjr3

Hall, K. L., Vogel, A. L., Huang, G. C., Serrano, K. J., Rice, E. L., Tsakraklides, S. P., \& Fiore, S. M. (2018). The science of team science: A review of the empirical evidence and research gaps on collaboration in science. American Psychologist, 73(4), 532-548. https://doi.org/10/gdrdqp 
Hardwicke, T. E., \& Ioannidis, J. P. A. (2018). Mapping the universe of registered reports. Nature Human Behaviour, 2(11), 793-796. https://doi.org/10/gf9dbb

Holcombe, A. O. (2019). Contributorship, not authorship: Use CRediT to indicate who did what. Publications, 7(3), 48. https://doi.org/10/gghnrr

Klein, R. A., Ratliff, K. A., Vianello, M., Adams, R. B., Bahník, Š., Bernstein, M. J., Bocian, K., Brandt, M. J., Brooks, B., Brumbaugh, C. C., Cemalcilar, Z., Chandler, J., Cheong, W., Davis, W. E., Devos, T., Eisner, M., Frankowska, N., Furrow, D., Galliani, E. M., ... Nosek, B. A. (2014). Investigating Variation in Replicability: A "Many Labs” Replication Project. Social Psychology, 45(3), 142-152. https://doi.org/10.1027/1864-9335/a000178

Klein, R. A., Vianello, M., Hasselman, F., Adams, B. G., Adams Jr, R. B., Alper, S., Aveyard, M., Axt, J. R., Babalola, M. T., \& Bahník, Š. (2018). Many Labs 2: Investigating variation in replicability across samples and settings. Advances in Methods and Practices in Psychological Science, 1(4), 443-490. https://doi.org/10.1177/2515245918810225

Lakens, D., Adolfi, F. G., Albers, C. J., Anvari, F., Apps, M. A. J., Argamon, S. E., Baguley, T., Becker, R. B., Benning, S. D., Bradford, D. E., Buchanan, E. M., Caldwell, A. R., Van Calster, B., Carlsson, R., Chen, S.-C., Chung, B., Colling, L. J., Collins, G. S., Crook, Z., ... Zwaan, R. A. (2018). Justify your alpha. Nature Human Behaviour, 2(3), 168-171. https://doi.org/10/gcz8f3

Many Primates, Altschul, D. M., Beran, M. J., Bohn, M., Call, J., DeTroy, S., Duguid, S. J., Egelkamp, C. L., Fichtel, C., Fischer, J., Flessert, M., Hanus, D., Haun, D. B. M., Haux, L. M., HernandezAguilar, R. A., Herrmann, E., Hopper, L. M., Joly, M., Kano, F., ... Watzek, J. (2019).

Establishing an infrastructure for collaboration in primate cognition research. PLOS ONE, 14(10), e0223675. https://doi.org/10/ggckgm

Moshontz, H., Campbell, L., Ebersole, C. R., IJzerman, H., Urry, H. L., Forscher, P. S., Grahe, J. E., McCarthy, R. J., Musser, E. D., \& Antfolk, J. (2018). The Psychological Science Accelerator: Advancing psychology through a distributed collaborative network. Advances in Methods and Practices in Psychological Science, 1(4), 501-515. https://doi.org/10.1177/2515245918797607 
Munafò, M. R., Nosek, B. A., Bishop, D. V. M., Button, K. S., Chambers, C. D., Percie du Sert, N., Simonsohn, U., Wagenmakers, E.-J., Ware, J. J., \& Ioannidis, J. P. A. (2017). A manifesto for reproducible science. Nature Human Behaviour, 1(1), 0021. https://doi.org/10/bw28

Newman, M. E. J. (2004). Coauthorship networks and patterns of scientific collaboration. Proceedings of the National Academy of Sciences, 101(Supplement 1), 5200-5205. https://doi.org/10/fgx363

Nosek, B. A., \& Bar-Anan, Y. (2012). Scientific utopia: I. Opening scientific communication. Psychological Inquiry, 23(3), 217-243. https://doi.org/10.1080/1047840X.2012.692215

Open Science Collaboration. (2012). An open, large-scale, collaborative effort to estimate the reproducibility of psychological science. Perspectives on Psychological Science, 7(6), 657-660. https://doi.org/10/f4d9dk

Open Science Collaboration. (2015). Estimating the reproducibility of psychological science. Science, 349(6251), aac4716-aac4716. https://doi.org/10.1126/science.aac4716

Petersen, A. M., Riccaboni, M., Stanley, H. E., \& Pammolli, F. (2012). Persistence and uncertainty in the academic career. Proceedings of the National Academy of Sciences, 109(14), 5213-5218. https://doi.org/10/f4k2vg

Sauermann, H., \& Haeussler, C. (2017). Authorship and contribution disclosures. Science Advances, 3(11), e1700404. https://doi.org/10/gcmbmc

Silberzahn, R., Uhlmann, E. L., Martin, D. P., Anselmi, P., Aust, F., Awtrey, E., Bahník, Š., Bai, F., Bannard, C., \& Bonnier, E. (2018). Many analysts, one data set: Making transparent how variations in analytic choices affect results. Advances in Methods and Practices in Psychological Science, 1(3), 337-356. https://doi.org/10.1177/2515245917747646

Smith, E., Williams-Jones, B., Master, Z., Larivière, V., Sugimoto, C. R., Paul-Hus, A., Shi, M., \& Resnik, D. B. (2019). Misconduct and Misbehavior Related to Authorship Disagreements in Collaborative Science. Science and Engineering Ethics. https://doi.org/10.1007/s11948-019$00112-4$

Uhlmann, E. L., Ebersole, C. R., Chartier, C. R., Errington, T. M., Kidwell, M. C., Lai, C. K., McCarthy, 
R. J., Riegelman, A., Silberzahn, R., \& Nosek, B. A. (2019). Scientific utopia III: Crowdsourcing science. Perspectives on Psychological Science, 14(5), 711-733. https://doi.org/10/gf4jhq

Wang, D., Yan, K.-K., Rozowsky, J., Pan, E., \& Gerstein, M. (2016). Temporal Dynamics of Collaborative Networks in Large Scientific Consortia. Trends in Genetics: TIG, 32(5), 251-253. https://doi.org/10/f8nm3w

Wuchty, S., Jones, B. F., \& Uzzi, B. (2007). The increasing dominance of teams in production of knowledge. Science, 316(5827), 1036-1039. https://doi.org/10/bb6p62 